(C) 2008, IEEE. Reprinted, with permission, from Aspin, R, An initial study into augmented inward looking exploration and navigation in CAVE-like IPT systems, IEEE Virtual Reality 08, 2008.

This material is posted here with permission of the IEEE. Such permission of the IEEE does not in any way imply IEEE endorsement of any of the University of Salford's products or services. Internal or personal use of this material is permitted. However, permission to reprint/republish this material for advertising or promotional purposes or for creating new collective works for resale or redistribution must be obtained from the IEEE by writing to pubs-permissions@ieee.org.

By choosing to view this document, you agree to all provisions of the copyright laws protecting it. 


\title{
An initial study into augmented inward looking exploration and navigation in CAVE-like IPT systems
}

\author{
Rob Aspin* \\ Centre for Virtual Environments \\ University of Salford
}

\begin{abstract}
This research presents and evaluates a CAVE-like IPT system in which a Tracked Display and Interaction Device (TDID), based on a Tablet PC, is used to display an augmented viewing system for detailed examination of a close focused objects. This maintains wider context, displayed on the IPT display environment, thereby enabling effective wayfinding and navigation, while still enabling detailed examination of the region of interest on a position sensitive high-resolution display (TDID). Evaluation against an unaugmented CAVE-like IPT system, demonstrates the effectiveness of this approach in enabling users to both explore around the model effectively resolve fine detail.
\end{abstract}

Index Terms: H.5.2 [Information Interfaces and Presentation]: User Interfaces-Input devices and strategies;

\section{INTRODUCTION}

Navigating and exploring small detailed structures in IPT systems is challenging [5] and a wide variety of approaches exist, particularly in the case of large scale IPT systems [4]. However, in small scale environments, where a user may be required to navigate round and explore a detailed, centred model from an inward looking perspective, the overlapping layers of detail and small scale present additional problems. If a user navigates to a focused region of interest surrounding landmarks essential for effective navigation are lost. Conversely by maintaining perception of the wider context users are unable to focus in on the fine detail they wish to view. While approaches to integrate both focus and context have been proposed and researched these are predominantly aimed at navigation of medium/large scale environments through which the user can travel, rather than smaller scale models which require inward looking exploration to navigate around the object of interest.

In our approach the immersive environment, displayed on the IPT system, is used to provide a contextual, peripheral view, while a tracked monoscopic display device is used to present a highresolution micro/macroscopic window responsive to the users location. This enables intuitive exploration, selection and manipulation of the detailed model, while maintaining peripheral context for the user to effectively navigate and orientate themselves in the wider environment. Comparative evaluation has been undertaken by examination of the manner in which this system improves exploration and navigation against that of a more traditional tracked joystick controller operating within the same immersive display system.

\section{BACKGROUND}

Bjrk suggests that it "might be important to give users access to both overview and detailed information at the same time" [1], enabing the object of primary interest to be presented in detail (focus) while having an overview (context) displayed at the same time.

*e-mail: R.Aspin@salford.ac.uk
Typically this has been enacted as a foveal insert to improve the localised viewing against a large scale depiction of massive $2 \mathrm{D}$ data sets. Staadt et al's [3] present a combined output from a pair of projectors on a single display surface compositing both foveal and peripheral views. Context display is presented by a single rear projection unit while a smaller front projector, equipped with a motorised pan-tilt mirror, is used to display a small, high resolution, overlay. Sanneblad [2], presents a tracked physical device held by the user. Provided this physical device is maintained in close proximity to the display screen, a high resolution representation of the displayed data it overlays is presented against the wider context. The fact that this approach operates in a tracked space, independent of the surrounding display system ideally matches the standard CAVE-like system configuration in which a tracked area is enclosed by the display screens, suggesting that an extension to viewing 3D data sets is possible. In our approach the user's head position is tracked and stereo imagery, presented on all four of the IPT display screens is corrected for the viewing position creating the impression of a holographic effect. Staadt's screen space solution would present a graphically inconstant insert, depth located against the screen; however Sanneblad's solution can be extended to the 3D CAVE-like display system by tracking both device and user's head position and presenting the 3D spatially corrected imagery onto the local vewing device.

\section{IMPLMENTATION}

Implementation was enacted using the University of Salford's Centre for Virtual Environment's (CVE) FLOW/ICE system [6], providing stereo visualisation and $3 \mathrm{D}$ interaction in a 4 sided CAVElike IPT system and a mono-display and interaction client (figure 1). 3D Tracking was enacted using the electromagnetic tracking system fitted in the IPT system. Sensors we attached to the user's head, tablet device and joystick device. View frustrums for the IPT screens were calculated using CAVELib based on the user's head position. When the Tablet PC was used as a aditional viewing device CAVELib was once again used to generate a view frustrum for the tablet device based on the user's head position and the tablet position and orientation (figure 2). In order to perform evalua-

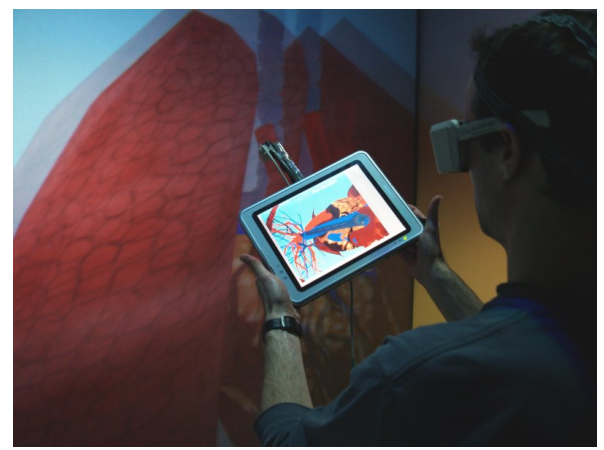

Figure 1: TDID System. 


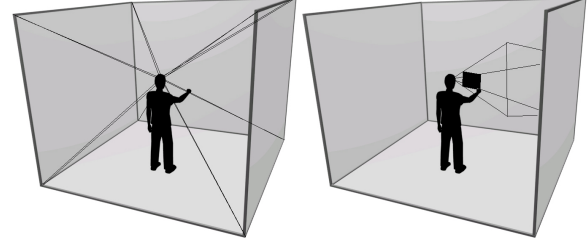

Figure 2: IPT System view frustrum (left) \& tablet view frustrum (right)

\begin{tabular}{cccc} 
Question & $\begin{array}{c}\text { Joystick Mean } \\
\text { (Variance) }\end{array}$ & $\begin{array}{c}\text { TDID Mean } \\
\text { (Variance) }\end{array}$ & $\begin{array}{c}\text { Poss result } \\
\text { by Chance }\end{array}$ \\
\hline & & & \\
$1 \& 5$ & $4.8(1.7)$ & $5.6(2.3)$ & 0.32 \\
$2 \& 6$ & $1.6(0.3)$ & $6.6(0.8)$ & $1.4 * 10^{-5}$ \\
$3 \& 7$ & $5.4(1.3)$ & $5.2(1.2)$ & 0.48 \\
$4 \& 8$ & $2.8(2.7)$ & $2.6(3.3)$ & 0.56
\end{tabular}

Table 1: Analysis of explicit evaluation results

tion experiments a test case of sufficient complexity and detail that was also small enough to be contained within the physical dimensions of the CAVE-like IPT system needed to be identified. These constraints ensured that large scale navigation did not become a major factor, instead requiring users to enact most navigation through body movement while still performing an element of virtual movement to reposition themseleves around the object. A search of existing models identified an animated model of the heart and lungs. This had been used for previous experiments with users suggesting that as they zoomed in on regions of interest it became difficult to maintain a concept of orientation against the wider context of the model.

\section{Discussion}

Trials were enacted in randomised order, with experienced users attempting the same task with both Tracked Display and Interaction Device (TDID) and tracked joystick controller. In the actual trial the model was placed at some distance from the user, who was then asked to decrease their distance to the model by navigating towards it, or selecting and manipulating the model position. Once positioned the user was asked to examine the model before modifying their position so that they were viewing the major blood vessel leaving the heart (from the inside) and to navigate from the heart, through to the lung, along the path of the blood vessel. Users' were observed during the trial and completed a questionnaire after each session.

Q1\&5: I was able to interact with the environment in an intuitive manner

Q2\&6: I could effectively select and manipulate objects in the environment

Q3\&7: I could easily navigate through the environment

Q4\&8: I easily became lost or disorientated in the environment

Questions 1 to 4 were asked of the user's experiences using the tracked joystick controller, while questions 5 to 8 were asked of the users' experience of achieving the same task using TDID system. This analysis (Table 1) shows that only in the case of question 2 , concerning the selection and manipulation of objects, is there a strong positive case for the use of the TDID approach over the use of the joystick controller. Anecdotal evidence, captured by observation, user comments and interview reinforces this, showing that not only did users' like the TDID approach, but that in some ways it created a more natural environment for them to explore the virtual environment.

None of the participants appeared to notice that the TDID system only presented the detailed view in a monoscopic form (participants were not told about this before the trial started). However, several participants did comment on how much more detail they could see using the tablet device, and how much more comfortable this was for them to explore the model as the un-augmented system's visual presentation was often hard to see, particularly when the user was close to the model, requiring significant concentration to bring the two stereo images into focus.

\section{ConClusion}

Explicit evaluation of this approach, compared to the use of a more traditional accepted intuitive input device, a 3D tracked joystick controller, shows that it performed as well as the more accepted device for most tasks, and better in the case of selection and manipulation of virtual objects.

Trial observations, and post trial discussions suggests that users' not only found the TDID system intuitive and easy to use, but also found the focus + context viewing mode that the system provided helped their navigation and exploration of the detailed model by preserving an understanding of the context while allowing fine grained movement at a detailed level. In cases of inward looking exploration, when the virtual object is contained within the physical dimensions of the larger display system, this system appears to improve the users' ability to resolve the detail of the object they are viewing while also allowing more natural movment around the object, thereby enabling users' to quickly transfer their focus from one region to the next without losing their sence of place in the environment.

With the tracked joystick controller most users navigated to a specific location to view the model, even though they could achieve the same viewing transformation by changing their body position within the CAVE-like IPT system. With the tracked interaction device, rather than navigating to a new viewing position within the virtual environment, most users simply moved their body position and that of the tablet device to change their viewpoint. This suggests that they found this a more intuitive method of examination, as they were using a more natural form of controlling their view transformation. In addition, users of the tracked interaction device would regularly move their physical view point to a position looking at one of the 'missing' screens on the IPT system and viewing the object solely on the tracked device without apparently noticing that the 'context' view was not present behind the tracked device. Both of these observations suggest that users were experiencing greater levels of immersion, and therefore a greater sense of presence, which once again re-enforces the suitability of the tracked interaction device for this type of application.

\section{REFERENCES}

[1] S. Bjrk and J. Redstrm. Redefining the focus and context of focus+context visualisation. In IEEE Symposium on Information Visualisation, pages 85-89, Salt Lake City, UT, USA, 2000. IEEE.

[2] J. Sanneblad and L. E. Holmquist. Ubiquitous graphics: combining hand-held and wall-size displays to interact with large images. In AVI '06: Proceedings of the working conference on Advanced visual interfaces, pages 373-377, New York, NY, USA, 2006. ACM Press.

[3] O. G. Staadt, B. A. Ahlborn, O. Kreylos, and B. Hamann. A foveal inset for large display environments. In Proceedings of the 2006 ACM international conference on Virtual reality continuum and its applications, pages 281-288. ACM Press, Hong Kong, China, 2006.

[4] D. S. Tan, D. Gergle, P. Scupelli, and R. Pausch. Physically large displays improve performance on spatial tasks. ACM Trans. Comput.Hum. Interact., 13(1):71-99, 2006.

[5] S. Vallance and P. Calder. Context in 3d planar navigation. In Proceedings of the 2nd Australasian conference on User interface, pages 93-99. IEEE Computer Society, Queensland, Australia, 2001.

[6] R. Wolff, D. Roberts, and O. Otto. Collaboration around shared objects in immersive virtual environments. In 8th IEEE International Symposium on Distributed Simulation and Real-Time Applications (DS-RT 2004), pages 206-209, 2004. 